\title{
Action Recognition by Dense Trajectories
}

\author{
Heng Wang ${ }^{\dagger} \quad$ Alexander Kläser ${ }^{\ddagger}$ \\ ${ }^{\dagger}$ National Laboratory of Pattern Recognition \\ Institute of Automation, Chinese Academy of Sciences
Cordelia Schmid ${ }^{\ddagger} \quad$ Cheng-Lin Liu ${ }^{\dagger}$
${ }^{\ddagger}$ LEAR, INRIA Grenoble, LJK \\ Grenoble, France \\ \{hwang, liucl\}@nlpr.ia.ac.cn \\ \{Alexander.Klaser, Cordelia.Schmid\}einria.fr
}

\begin{abstract}
Feature trajectories have shown to be efficient for representing videos. Typically, they are extracted using the KLT tracker or matching SIFT descriptors between frames. However, the quality as well as quantity of these trajectories is often not sufficient. Inspired by the recent success of dense sampling in image classification, we propose an approach to describe videos by dense trajectories. We sample dense points from each frame and track them based on displacement information from a dense optical flow field. Given a state-of-the-art optical flow algorithm, our trajectories are robust to fast irregular motions as well as shot boundaries. Additionally, dense trajectories cover the motion information in videos well.

We, also, investigate how to design descriptors to encode the trajectory information. We introduce a novel descriptor based on motion boundary histograms, which is robust to camera motion. This descriptor consistently outperforms other state-of-the-art descriptors, in particular in uncontrolled realistic videos. We evaluate our video description in the context of action classification with a bag-of-features approach. Experimental results show a significant improvement over the state of the art on four datasets of varying difficulty, i.e. KTH, YouTube, Hollywood2 and UCF sports.
\end{abstract}

\section{Introduction}

Local features are a popular way for representing videos. They achieve state-of-the-art results for action classification when combined with a bag-of-features representation. Recently, interest point detectors and local descriptors have been extended from images to videos. Laptev and Lindeberg [13] introduced space-time interest points by extending the Harris detector. Other interest point detectors include detectors based on Gabor filters $[1,5]$ or on the determinant of the spatio-temporal Hessian matrix [33]. Feature descriptors range from higher order derivatives (local jets), gradient information, optical flow, and brightness information $[5,14,24]$ to spatio-temporal extensions of image

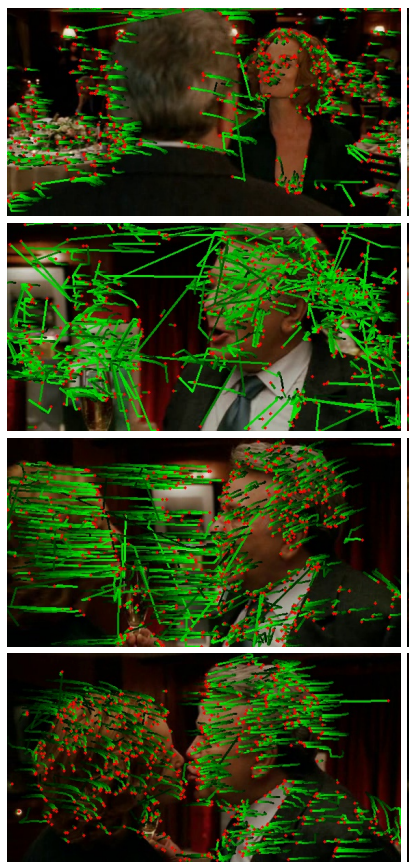

KLT

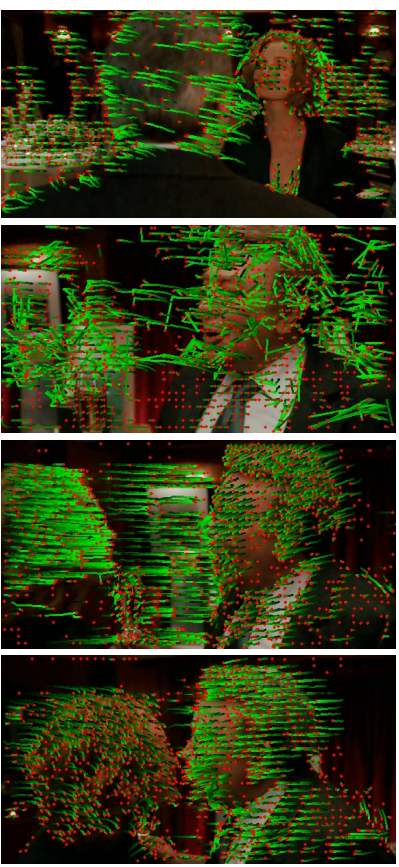

Dense trajectories
Figure 1. A comparison of the KLT tracker and dense trajectories. Red dots indicate the point positions in the current frame. Dense trajectories are more robust to irregular abrupt motions, in particular at shot boundaries (second row), and capture more accurately complex motion patterns.

descriptors, such as 3D-SIFT [25], HOG3D [11], extended SURF [33], or Local Trinary Patterns [34].

However, the 2D space domain and 1D time domain in videos have very different characteristics. It is, therefore, intuitive to handle them in a different manner than via interest point detection in a joint 3D space. Tracking interest points through video sequences is a straightforward choice. Some recent methods [20, 21, 27] show impressive results for action recognition by leveraging the motion information of trajectories. Messing et al. [21] extracted feature trajectories by tracking Harris3D interest points [13] with the KLT tracker [18]. Trajectories are represented as sequences of log-polar quantized velocities. Matikainen et al. [20] used 


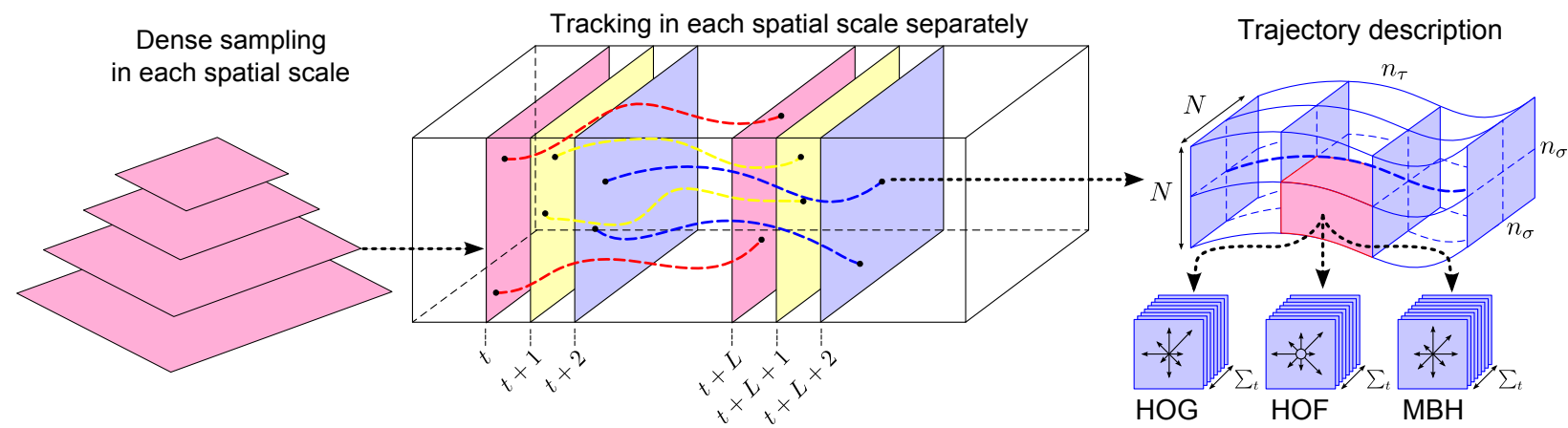

Figure 2. Illustration of our dense trajectory description. Left: Feature points are sampled densely for multiple spatial scales. Middle: Tracking is performed in the corresponding spatial scale over $L$ frames. Right: Trajectory descriptors are based on its shape represented by relative point coordinates as well as appearance and motion information over a local neighborhood of $N \times N$ pixels along the trajectory. In order to capture the structure information, the trajectory neighborhood is divided into a spatio-temporal grid of size $n_{\sigma} \times n_{\sigma} \times n_{\tau}$.

a standard KLT tracker. Trajectories in a video are clustered, and an affine transformation matrix is computed for each cluster center. The elements of the matrix are used to represent the trajectories. Sun et al. [27] extracted trajectories by matching SIFT descriptors between two consecutive frames. They imposed a unique-match constraint among the descriptors and discarded matches that are too far apart.

Dense sampling has shown to improve results over sparse interest points for image classification [7, 22]. The same has been observed for action recognition in a recent evaluation by Wang et al. [32], where dense sampling at regular positions in space and time outperforms state-of-the-art space-time interest point detectors. In contrast, trajectories are often obtained by the KLT tracker, which is designed to track sparse interest points [18]. Matching dense SIFT descriptors is computationally very expensive [15] and, thus, infeasible for large video datasets.

In this paper, we propose an efficient way to extract dense trajectories. The trajectories are obtained by tracking densely sampled points using optical flow fields. The number of tracked points can be scaled up easily, as dense flow fields are already computed. Furthermore, global smoothness constraints are imposed among the points in dense optical flow fields, which results in more robust trajectories than tracking or matching points separately, see Figure 1. Dense trajectories have not been employed previously for action recognition. Sundaram et al. [28] accelerated dense trajectories computation on a GPU. Brox et al. [2] segmented objects by clustering dense trajectories. A similar approach is used in [17] for video object extraction.

Motion is the most informative cue for action recognition. It can be due to the action of interest, but also be caused by background or the camera motion. This is inevitable when dealing with realistic actions in uncontrolled settings. How to separate action motion from irrelevant motion is still an open problem. Ikizler-Cinbis et al. [9] applied video stabilization via a motion compensation procedure, where most camera motion is removed. Uemura et al. [30] segmented feature tracks to separate the motion characterizing the actions from the dominant camera motion.

To overcome the problem of camera motion, we introduce a local descriptor that focuses on foreground motion. Our descriptor extends the motion coding scheme based on motion boundaries developed in the context of human detection [4] to dense trajectories. We show that motion boundaries encoded along the trajectories significantly outperform state-of-the-art descriptors.

This paper is organized as follows. In section 2, we introduce the approach for extracting dense trajectories. We, then, show how to encode feature descriptors along the trajectories in section 3. Finally, we present the experimental setup and discuss the results in sections 4 and 5 respectively. The code to compute dense trajectories and their description is available online ${ }^{1}$.

\section{Dense trajectories}

Dense trajectories are extracted for multiple spatial scales, see Figure 2. Feature points are sampled on a grid spaced by $W$ pixels and tracked in each scale separately. Experimentally, we observed that a sampling step size of $W=5$ is dense enough to give good results. We used 8 spatial scales spaced by a factor of $1 / \sqrt{2}$. Each point $P_{t}=\left(x_{t}, y_{t}\right)$ at frame $t$ is tracked to the next frame $t+1$ by median filtering in a dense optical flow field $\omega=\left(u_{t}, v_{t}\right)$.

$$
P_{t+1}=\left(x_{t+1}, y_{t+1}\right)=\left(x_{t}, y_{t}\right)+\left.(M * \omega)\right|_{\left(\bar{x}_{t}, \bar{y}_{t}\right)},
$$

where $M$ is the median filtering kernel, and $\left(\bar{x}_{t}, \bar{y}_{t}\right)$ is the rounded position of $\left(x_{t}, y_{t}\right)$. This is more robust than bilinear interpolation used in [28], especially for points near motion boundaries. Once the dense optical flow field is computed, points can be tracked very densely without additional cost. Points of subsequent frames are concatenated to form a trajectory: $\left(P_{t}, P_{t+1}, P_{t+2}, \ldots\right)$. To extract dense

\footnotetext{
${ }^{1}$ http://lear.inrialpes.fr/software
} 


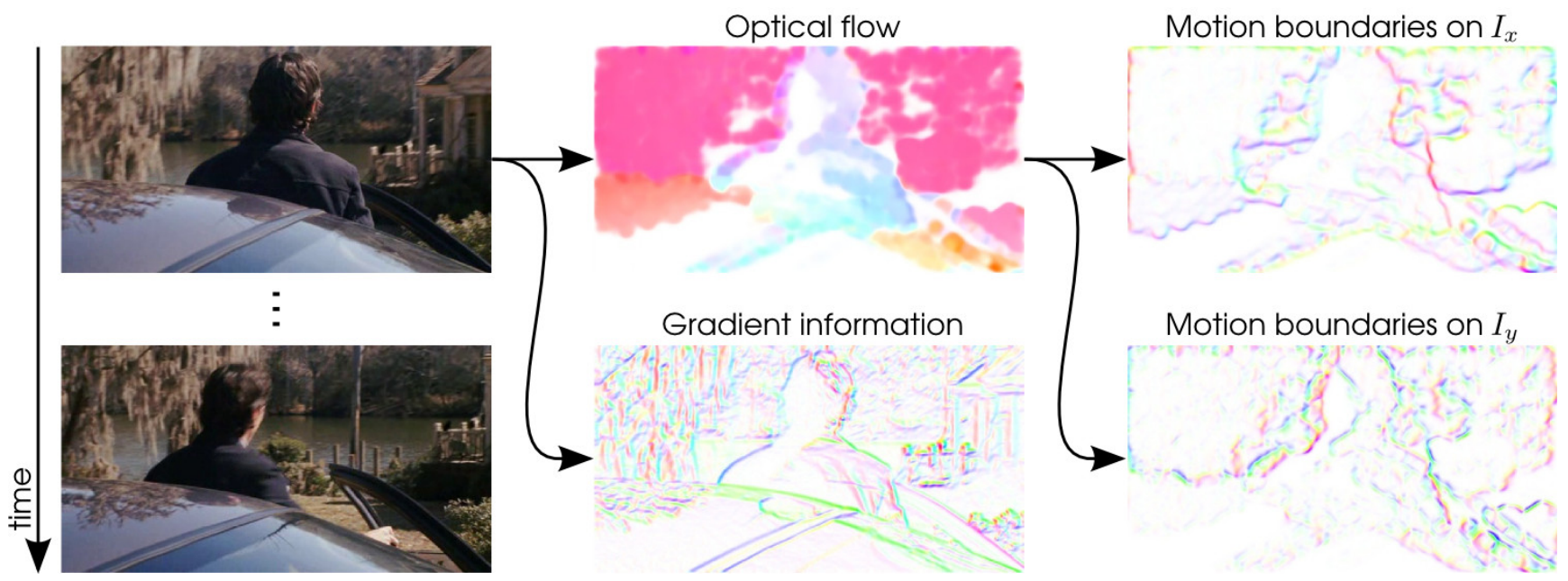

Figure 3. Illustration of the information captured by HOG, HOF, and MBH descriptors. For each image, gradient/flow orientation is indicated by color (hue) and magnitude by saturation. Motion boundaries are computed as gradients of the $\mathrm{x}$ and y optical flow components separately. Compared to optical flow, motion boundaries suppress most camera motion in the background and highlight the foreground motion. Unlike gradient information, motion boundaries eliminate most texture information from the static background.

optical flow, we use the algorithm by Färneback [6] as implemented in the OpenCV library². We found this algorithm to be a good compromise between accuracy and speed.

A common problem in tracking is drifting. Trajectories tend to drift from their initial location during tracking. To avoid this problem, we limit the length of a trajectory to $L$ frames. As soon as a trajectory exceeds length $L$, it is removed from the tracking process, see Figure 2 (middle). To assure a dense coverage of the video, we verify the presence of a track on our dense grid in every frame. If no tracked point is found in a $W \times W$ neighborhood, this feature point is sampled and added to the tracking process. Experimentally, we chose a trajectory length of $L=15$ frames.

In homogeneous image areas without any structure, it is impossible to track points. Here, we use the same criterion as Shi and Tomasi [26]. When a feature point is sampled, we check the smaller eigenvalue of its autocorrelation matrix. If it is below a threshold, this point will not be included in the tracking process. Since for action recognition we are mainly interested in dynamic information, static trajectories are pruned in a pre-processing stage. Trajectories with sudden large displacements, most likely to be erroneous, are also removed. Figure 1 compares dense and KLT trajectories. We can observe that dense trajectories are more robust and denser than the trajectories obtained by the KLT tracker.

The shape of a trajectory encodes local motion patterns. Given a trajectory of length $L$, we describe its shape by a sequence $S=\left(\Delta P_{t}, \ldots, \Delta P_{t+L-1}\right)$ of displacement vectors $\Delta P_{t}=\left(P_{t+1}-P_{t}\right)=\left(x_{t+1}-x_{t}, y_{t+1}-y_{t}\right)$. The resulting vector is normalized by the sum of the magnitudes

\footnotetext{
${ }^{2}$ http://opencv.willowgarage.com/wiki/
}

of the displacement vectors:

$$
S^{\prime}=\frac{\left(\Delta P_{t}, \ldots, \Delta P_{t+L-1}\right)}{\sum_{j=t}^{t+L-1}\left\|\Delta P_{j}\right\|}
$$

We refer to this vector by trajectory descriptor. We have also evaluated representing trajectories at multiple temporal scales, in order to recognize actions with different speeds. However, this did not improve the results in practice. Therefore, we use trajectories with a fixed length $L$ in our experiments.

\section{Trajectory-aligned descriptors}

Local descriptors computed in a 3D video volume around interest points have become a popular way for video representation $[5,11,14,25,33]$. To leverage the motion information in our dense trajectories, we compute descriptors within a space-time volume around the trajectory, see Figure 2 (right). The size of the volume is $N \times N$ pixels and $L$ frames. To embed structure information in the representation, the volume is subdivided into a spatio-temporal grid of size $n_{\sigma} \times n_{\sigma} \times n_{\tau}$. The default parameters for our experiments are $N=32, n_{\sigma}=2, n_{\tau}=3$, which has shown to be optimal based on cross validation on the training set of the Hollywood2. We give results using different parameter settings in section 5.3.

Among the existing descriptors for action recognition, HOGHOF [14] has shown to give excellent results on a variety of datasets [32]. HOG (histograms of oriented gradients) [3] focuses on static appearance information, whereas HOF (histograms of optical flow) captures the local motion information. We compute HOGHOF along our dense trajectories. For both HOG and HOF, orientations are quantized into 8 bins using full orientations, with an additional zero 

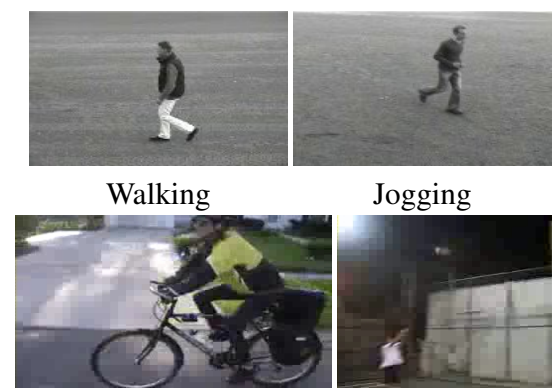

Biking

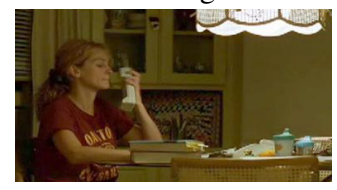

AnswerPhone

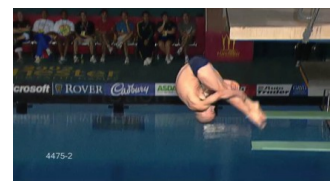

Diving

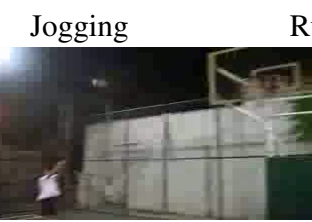

Shooting

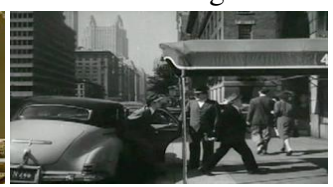

GetOutCar

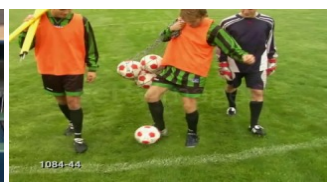

Kicking

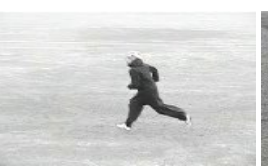

Running

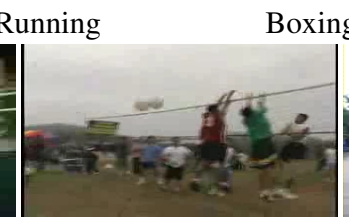

Spiking

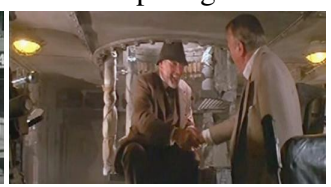

HandShake

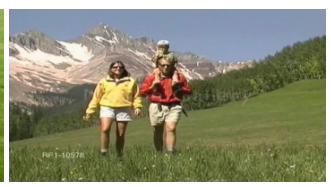

Walking

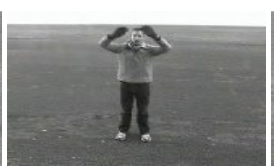

Waving

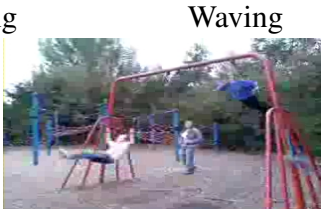

Swinging

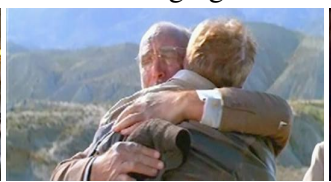

HugPerson

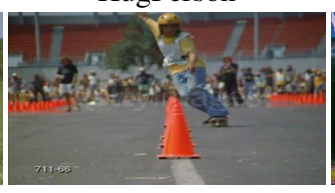

Skateboarding

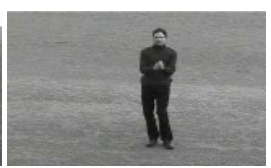

Clapping

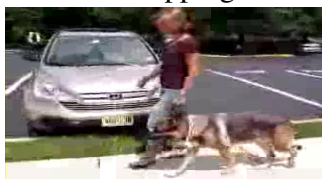

Walking dog

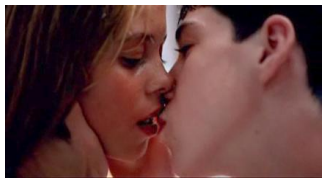

Kiss

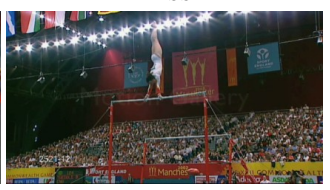

High-Bar-Swinging

Figure 4. Sample frames from video sequences of KTH (first row), YouTube (second row), Hollywood2 (third row) and UCF sports (last row) action datasets.

bin for HOF (i.e., in total 9 bins). Both descriptors are normalized with their $L_{2}$ norm. Figure 3 shows a visualization of HOGHOF.

Optical flow computes the absolute motion, which inevitably includes camera motion [9]. Dalal et al. [4] proposed the MBH (motion boundary histogram) descriptor for human detection, where derivatives are computed separately for the horizontal and vertical components of the optical flow. This descriptor encodes the relative motion between pixels, as shown in Figure 3. Here we use MBH to describe our dense trajectories.

The MBH descriptor separates the optical flow field $I_{\omega}=\left(I_{x}, I_{y}\right)$ into its $x$ and $y$ component. Spatial derivatives are computed for each of them and orientation information is quantized into histograms, similarly to the HOG descriptor. We obtain an 8-bin histogram for each component, and normalize them separately with the $L_{2}$ norm. Since MBH represents the gradient of the optical flow, constant motion information is suppressed and only information about changes in the flow field (i.e., motion boundaries) is kept. Compared to video stabilization [9] and motion compensation [30], this is a simple way to eliminate noise due to background motion. This descriptor yields excellent results when combined with our dense trajectories. For instance, on the YouTube dataset [16], MBH significantly outperforms HOF, see section 5.

For both HOF and MBH descriptors, we reuse the dense optical flow that is already computed to extract dense trajectories. This makes our feature computation process very efficient.

\section{Experimental setup}

In this section, we first describe the datasets used for action recognition. We, then, briefly present the bag-offeatures model used for evaluating our dense trajectory features as well as the KTL tracking baseline.

\subsection{Datasets}

Our dense trajectories are extensively evaluated on four standard action datasets: KTH, YouTube, Hollywood2, and UCF sports, see Figure 4. These datasets are very diverse. The KTH dataset views actions in front of a uniform background, whereas the Hollywood2 dataset contains real movies with significant background clutter. The YouTube videos are low quality, whereas UCF sport videos are high resolution.

The KTH dataset $[24]^{3}$ consists of six human action classes: walking, jogging, running, boxing, waving and clapping. Each action is performed several times by 25 subjects. The sequences were recorded in four different scenarios: outdoors, outdoors with scale variation, outdoors with different clothes and indoors. The background is homogeneous and static in most sequences. In total, the data consists of 2391 video samples. We follow the original experimental setup of the authors, e.g., divide the samples into test set (9 subjects: 2, 3, 5, 6, 7, 8, 9, 10, and 22) and training set (the remaining 16 subjects). As in the initial paper [24], we train and evaluate a multi-class classifier and report average accuracy over all classes as performance measure.

\footnotetext{
${ }^{3}$ http://www.nada.kth.se/cvap/actions/
} 
The YouTube dataset $[16]^{4}$ contains 11 action categories: basketball shooting, biking/cycling, diving, golf swinging, horse back riding, soccer juggling, swinging, tennis swinging, trampoline jumping, volleyball spiking, and walking with a dog. This dataset is challenging due to large variations in camera motion, object appearance and pose, object scale, viewpoint, cluttered background and illumination conditions. The dataset contains a total of 1168 sequences. We follow the original setup [16] using leave one out cross validation for a pre-defined set of 25 folds. Average accuracy over all classes is reported as performance measure.

The Hollywood2 dataset $[19]^{5}$ has been collected from 69 different Hollywood movies. There are 12 action classes: answering the phone, driving car, eating, fighting, getting out of car, hand shaking, hugging, kissing, running, sitting down, sitting up, and standing up. In our experiments, we used the clean training dataset. In total, there are 1707 action samples divided into a training set (823 sequences) and a test set ( 884 sequences). Train and test sequences come from different movies. The performance is evaluated by computing the average precision (AP) for each of the action classes and reporting the mean AP over all classes (mAP) as in [19].

The UCF sport dataset $[23]^{6}$ contains ten human actions: swinging (on the pommel horse and on the floor), diving, kicking (a ball), weight-lifting, horse-riding, running, skateboarding, swinging (at the high bar), golf swinging and walking. The dataset consists of 150 video samples which show a large intra-class variability. To increase the amount of data samples, we extend the dataset by adding a horizontally flipped version of each sequence to the dataset. Similar to the KTH actions dataset, we train a multi-class classifier and report the average accuracy over all classes. We use a leave-one-out setup and test on each original sequence while training on all other sequences together with their flipped versions (i.e., the flipped version of the tested sequence is removed from the training set).

\subsection{Bag of features}

To evaluate the performance of our dense trajectories, we use a standard bag-of-features approach. We first construct a codebook for each descriptor (trajectory, HOG, HOF, $\mathrm{MBH}$ ) separately. We fix the number of visual words per descriptor to 4000 which has shown to empirically give good results for a wide range of datasets. To limit the complexity, we cluster a subset of 100,000 randomly selected training features using $k$-means. To increase precision, we initialize $k$-means 8 times and keep the result with the lowest error.

\footnotetext{
${ }^{4}$ http://www.cs.ucf.edu/ Iiujg/YouTube\_Action \ _dataset.html

${ }^{5}$ http://lear.inrialpes.fr/data

${ }^{6}$ http://www.cs.ucf.edu/vision/public_html/
}

Descriptors are assigned to their closest vocabulary word using Euclidean distance. The resulting histograms of visual word occurrences are used as video descriptors.

For classification we use a non-linear SVM with a $\chi^{2}$ kernel [14]. Different descriptors are combined in a multichannel approach as in [31]:

$$
K\left(x_{i}, x_{j}\right)=\exp \left(-\sum_{c} \frac{1}{A^{c}} D\left(x_{i}^{c}, x_{j}^{c}\right)\right),
$$

where $D\left(x_{i}^{c}, x_{j}^{c}\right)$ is the $\chi^{2}$ distance between video $x_{i}$ and $x_{j}$ with respect to the $c$-th channel. $A^{c}$ is the mean value of $\chi^{2}$ distances between the training samples for the $c$-th channel [36]. In the case of multi-class classification, we use a one-against-rest approach and select the class with the highest score.

\subsection{Baseline KLT trajectories}

To compare our dense trajectories with the standard KLT tracker [18], we use the implementation of the KLT tracker from OpenCV. In each frame 100 interest points are detected, and added to the tracker, which is somewhat denser than space-time interest points [32]. Interest points are tracked through the video for $L$ frames. This is identical to the procedure used for our dense trajectories. We also use the same descriptors for the KLT trajectories, e.g. the trajectory shape is represented by normalized relative point coordinates, and HOG, HOF, MBH descriptors are extracted around the trajectories.

\section{Experimental results}

In this section, we evaluate the performance of our description and compare to state-of-the-art methods. We also determine the influence of different parameter settings.

\subsection{Evaluation of our dense trajectory descriptors}

In this section we compare dense and KLT trajectories as well as the different descriptors. We use our default parameters for this comparison. To compute the descriptors, we set $N=32, n_{\sigma}=2, n_{\tau}=3$ for both baseline KLT and dense trajectories. We fix the trajectory length to $L=15$, and the dense sampling step size to $W=5$.

Results for the four datasets are presented in Table 1. Overall, our dense trajectories outperform the KLT trajectories by $2 \%$ to $6 \%$. Since the descriptors are identical, this demonstrates that our dense trajectories describe the video structures more accurately.

Trajectory descriptors, which only describe the motion of the trajectories, give surprisingly good results by themselves, e.g. 90.2\% on KTH and $47.7 \%$ on Hollywood 2 for dense trajectories. This confirms the importance of motion information contained in the local trajectory patterns. We report only $67.2 \%$ on YouTube because the trajectory 


\begin{tabular}{|l||cc|cc|cc|cc|}
\hline & KLT & $\begin{array}{c}\text { KTH } \\
\text { Dense trajectories }\end{array}$ & KLT & $\begin{array}{c}\text { YouTube } \\
\text { Dense trajectories }\end{array}$ & KLT & $\begin{array}{c}\text { Hollywood2 } \\
\text { Dense trajectories }\end{array}$ & $\begin{array}{c}\text { UCF sports } \\
\text { Kense trajectories }\end{array}$ \\
\hline \hline Trajectory & $88.4 \%$ & $90.2 \%$ & $58.2 \%$ & $67.2 \%$ & $46.2 \%$ & $47.7 \%$ & $72.8 \%$ & $75.2 \%$ \\
HOG & $84.0 \%$ & $86.5 \%$ & $71.0 \%$ & $74.5 \%$ & $41.0 \%$ & $41.5 \%$ & $80.2 \%$ & $\mathbf{8 3 . 8 \%}$ \\
HOF & $92.4 \%$ & $93.2 \%$ & $64.1 \%$ & $72.8 \%$ & $48.4 \%$ & $50.8 \%$ & $72.7 \%$ & $77.6 \%$ \\
MBH & $93.4 \%$ & $\mathbf{9 5 . 0 \%}$ & $72.9 \%$ & $\mathbf{8 3 . 9 \%}$ & $48.6 \%$ & $\mathbf{5 4 . 2 \%}$ & $78.4 \%$ & $\mathbf{8 4 . 8 \%}$ \\
\hline Combined & $\mathbf{9 3 . 4 \%}$ & $\mathbf{9 4 . 2 \%}$ & $\mathbf{7 9 . 9 \%}$ & $\mathbf{8 4 . 2 \%}$ & $\mathbf{5 4 . 6 \%}$ & $\mathbf{5 8 . 3 \%}$ & $82.1 \%$ & $\mathbf{8 8 . 2 \%}$ \\
\hline
\end{tabular}

Table 1. Comparison of KLT and dense trajectories as well as different descriptors on KTH, YouTube, Hollywood2 and UCF sports. We report average accuracy over all classes for KTH, YouTube and UCF sports and mean AP over all classes for Hollywood2.

\begin{tabular}{|c|c|c|c|c|c|c|c|}
\hline \multicolumn{2}{|l|}{ KTH } & \multicolumn{2}{|l|}{ YouTube } & \multicolumn{2}{|c|}{ Hollywood2 } & \multicolumn{2}{|l|}{ UCF sports } \\
\hline Laptev et al. [14] & $91.8 \%$ & Liu et al. [16] & $71.2 \%$ & Wang et al. [32] & $47.7 \%$ & Wang et al. [32] & $85.6 \%$ \\
\hline Yuan et al. [35] & $93.3 \%$ & Ikizler-Cinbis et al. [9] & $75.21 \%$ & Gilbert et al. [8] & $50.9 \%$ & Kovashka et al. [12] & $87.27 \%$ \\
\hline Gilbert et al. [8] & $94.5 \%$ & & & Ullah et al. [31] & $53.2 \%$ & Kläser et al. [10] & $86.7 \%$ \\
\hline Kovashka et al. [12] & $94.53 \%$ & & & Taylor et al. [29] & $46.6 \%$ & & \\
\hline Our method & $94.2 \%$ & Our method & $84.2 \%$ & Our method & $58.3 \%$ & Our method & $88.2 \%$ \\
\hline
\end{tabular}

Table 2. Comparison of our dense trajectories characterized by our combined descriptor (Trajectory+HOG+HOF+MBH) with state-of-theart methods in the literature.

descriptors capture lots of motions from camera. Generally, HOF outperforms HOG as motion is more discriminative than static appearance for action recognition. However, HOG gets better results both on YouTube and UCF sports. The HOF descriptors computed on YouTube videos are heavily polluted by camera motions, since many videos are collected by hand-held cameras. Static scene context is very important for UCF sports actions which often involve specific equipment and scene types. MBH consistently outperforms the other descriptors on all four datasets. The improvement is most significant on the uncontrolled realistic datasets YouTube and Hollywood2. For instance, MBH is $11.1 \%$ better than HOF on YouTube. This confirms the advantage of suppressing background motion when dealing with optical flow.

\subsection{Comparison to the state of the art}

Table 2 compares our results to state of the art. On KTH, we obtain $94.2 \%$ which is comparable to the state of the art, i.e., $94.53 \%$ [12]. Note that on this dataset several authors use a leave-one-out cross-validation setting. Here, we only compare to those using the standard setting [24]. Interestingly, MBH alone obtains a slightly better performance on $\mathrm{KTH}$, i.e., $95.0 \%$, than combining all the descriptors together. Ullah et al. [31] also found that a combination of descriptors performed worse than a subset of them. On YouTube, we significantly outperform the current state-ofthe-art method [9] by $9 \%$, where video stabilization is used to remove camera motion. We report $58.3 \%$ on Hollywood 2 which is an improvement of $5 \%$ over [31]. Note that Ullah et al. [31] achieved better results by using additional images collected from Internet. The difference between all methods is rather small on UCF sports, which is largely due to the leave-one-out setting, e.g. 149 videos are used for training and only one for testing. Nevertheless, we outperform the state of the art [12] by $1 \%$.

We also compare the results per action class for YouTube and Hollywood2. On YouTube, our dense trajectories give best results for 8 out of 11 action classes when compare with the KLT baseline and the approach of [9], see Table 3. On Hollywood2, we compare the AP of each action class with the KLT baseline and the approach of [31], i.e., a combination of 24 spatio-temporal grids, see Table 4 . Our dense trajectories yield best results for 8 out of 12 action classes.

\begin{tabular}{|c||ccc|}
\hline & KLT & Dense trajectories & Ikizler-Cinbis [9] \\
\hline \hline b_shoot & $34.0 \%$ & $43.0 \%$ & $\mathbf{4 8 . 4 8 \%}$ \\
bike & $87.6 \%$ & $\mathbf{9 1 . 7 \%}$ & $75.17 \%$ \\
dive & $\mathbf{9 9 . 0 \%}$ & $\mathbf{9 9 . 0 \%}$ & $95.0 \%$ \\
golf & $95.0 \%$ & $\mathbf{9 7 . 0 \%}$ & $95.0 \%$ \\
h_ride & $76.0 \%$ & $\mathbf{8 5 . 0 \%}$ & $73.0 \%$ \\
s_juggle & $65.0 \%$ & $\mathbf{7 6 . 0 \%}$ & $53.0 \%$ \\
swing & $86.0 \%$ & $\mathbf{8 8 . 0 \%}$ & $66.0 \%$ \\
t_swing & $71.0 \%$ & $71.0 \%$ & $\mathbf{7 7 . 0 \%}$ \\
t_jump & $93.0 \%$ & $\mathbf{9 4 . 0 \%}$ & $93.0 \%$ \\
v_spike & $\mathbf{9 6 . 0 \%}$ & $95.0 \%$ & $85.0 \%$ \\
walk & $76.4 \%$ & $\mathbf{8 7 . 0 \%}$ & $66.67 \%$ \\
\hline Accuracy & $79.9 \%$ & $\mathbf{8 4 . 2 \%}$ & $75.21 \%$ \\
\hline
\end{tabular}

Table 3. Accuracy per action class for the YouTube dataset. We compare with the results reported in [9].

\begin{tabular}{|c||ccc|}
\hline & KLT & Dense trajectories & Ullah [31] \\
\hline \hline AnswerPhone & $18.3 \%$ & $\mathbf{3 2 . 6 \%}$ & $25.9 \%$ \\
DriveCar & $\mathbf{8 8 . 8 \%}$ & $88.0 \%$ & $85.9 \%$ \\
Eat & $\mathbf{7 3 . 4 \%}$ & $65.2 \%$ & $56.4 \%$ \\
FightPerson & $74.2 \%$ & $\mathbf{8 1 . 4 \%}$ & $74.9 \%$ \\
GetOutCar & $47.9 \%$ & $\mathbf{5 2 . 7 \%}$ & $44.0 \%$ \\
HandShake & $18.4 \%$ & $29.6 \%$ & $\mathbf{2 9 . 7 \%}$ \\
HugPerson & $42.6 \%$ & $\mathbf{5 4 . 2 \%}$ & $46.1 \%$ \\
Kiss & $65.0 \%$ & $\mathbf{6 5 . 8 \%}$ & $55.0 \%$ \\
Run & $76.3 \%$ & $\mathbf{8 2 . 1 \%}$ & $69.4 \%$ \\
SitDown & $59.0 \%$ & $\mathbf{6 2 . 5 \%}$ & $58.9 \%$ \\
SitUp & $\mathbf{2 7 . 7 \%}$ & $20.0 \%$ & $18.4 \%$ \\
StandUp & $63.4 \%$ & $\mathbf{6 5 . 2 \%}$ & $57.4 \%$ \\
\hline mAP & 54.6 & $\mathbf{5 8 . 3 \%}$ & $51.8 \%$ \\
\hline
\end{tabular}

Table 4. Average precision per action class for the Hollywood 2 dataset. We compare with the results reported in [31]. 

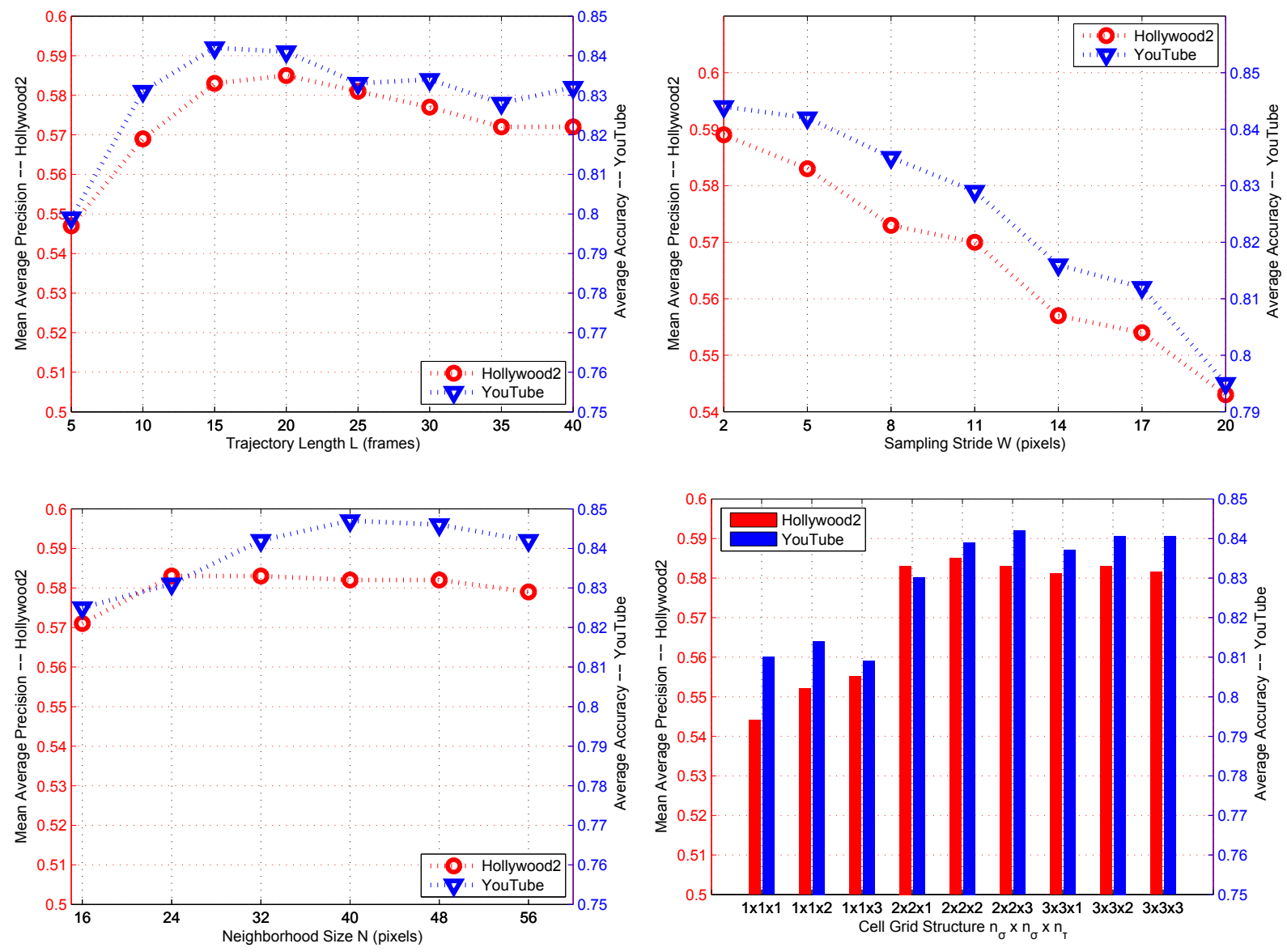

Figure 5. Results for different parameter settings on the Hollywood2 and YouTube datasets.

\subsection{Evaluation of trajectory parameters}

To evaluate the different parameter settings for dense trajectories, we report results on YouTube and Hollywood2, as they are larger and more challenging than the other two. We study the impact of the trajectory length, sampling step size, neighborhood size and cell grid structure. We evaluate the performance for a parameter at the time. The other parameters are fixed to the default values, i.e., trajectory length $L=15$, sampling step size $W=5$, neighborhood size $N=32$ and cell grid structure $n_{\sigma}=2, n_{\tau}=3$.

Figure 5 (top, left) evaluates the impact of the trajectory length $L$. For both datasets an increase of length $L$ improves performance up to a certain point ( $L=15$ or 20 ), and then decreases slightly, since longer trajectories have a higher chance to drift from the initial position. We achieve the best results with a trajectory length of 15 or 20 frames.

With respect to the sampling step size $W$, Figure 5 (top, right) shows that dense sampling improves the results as the step size decreases. This is consistent with dense sampling at regular positions [32], where more features in general improve the results. We report $58.9 \%$ (58.3\%) on Hollywood 2 and $84.4 \%(84.2 \%)$ on YouTube for a step size of 2 (5) pix- els. A sampling step of 2 pixels is extremely dense, i.e., every other pixel is sampled, and does not justify the minor gain obtained.

The results are relatively stable with regard to the neighborhood size $N$, see Figure 5 (bottom left). On Hollywood2, results are almost the same when $N$ changes from 24 pixels to 48 pixels. The best result on YouTube is $84.7 \%$ with a neighborhood size of 40 pixels. Dividing the video volume into cells improves the results on both Hollywood2 and YouTube. In particular, the performance increases significantly when the spatial cell grid $n_{\sigma}$ is increased from 1 to 2, see Figure 5 (bottom right). However, further increasing the number of cells, i.e., beyond $n_{\sigma}=2, n_{\tau}=3$, does not improve the results.

\section{Conclusions}

This paper has introduced an approach to model videos by combining dense sampling with feature tracking. Our dense trajectories are more robust than previous video descriptions. They capture the motion information in the videos efficiently and show improved performance over state-of-the-art approaches for action classification. We 
have also introduced an efficient solution to remove camera motion by computing motion boundaries descriptors along the dense trajectories. This successfully segments the relevant motion from background motion, and outperforms previous video stabilization methods. Our descriptors combine trajectory shape, appearance, and motion information. Such a representation has shown to be efficient for action classification, but could also be used in other areas, such as action localization and video retrieval.

Acknowledgments. This work was partly supported by National Natural Science Foundation of China (NSFC) under grant no.60825301 as well as the joint Microsoft/INRIA project and the European integrated project AXES.

\section{References}

[1] M. Bregonzio, S. Gong, and T. Xiang. Recognising action as clouds of space-time interest points. In CVPR, 2009.

[2] T. Brox and J. Malik. Object segmentation by long term analysis of point trajectories. In ECCV, 2010.

[3] N. Dalal and B. Triggs. Histograms of oriented gradients for human detection. In $C V P R, 2005$.

[4] N. Dalal, B. Triggs, and C. Schmid. Human detection using oriented histograms of flow and appearance. In ECCV, 2006.

[5] P. Dollár, V. Rabaud, G. Cottrell, and S. Belongie. Behavior recognition via sparse spatio-temporal features. In VS-PETS, 2005.

[6] G. Farnebäck. Two-frame motion estimation based on polynomial expansion. In Scandinavian Conference on Image Analysis, 2003.

[7] L. Fei-Fei and P. Perona. A Bayesian hierarchical model for learning natural scene categories. In CVPR, 2005.

[8] A. Gilbert, J. Illingworth, and R.Bowden. Action recognition using mined hierarchical compound features. IEEE PAMI, 2011.

[9] N. Ikizler-Cinbis and S. Sclaroff. Object, scene and actions: Combining multiple features for human action recognition. In $E C C V, 2010$.

[10] A. Kläser, M. Marszałek, I. Laptev, and C. Schmid. Will person detection help bag-of-features action recognition? In Technical Report, INRIA Grenoble - Rhone-Alpes, 2010.

[11] A. Kläser, M. Marszałek, and C. Schmid. A spatio-temporal descriptor based on 3D-gradients. In BMVC, 2008.

[12] A. Kovashka and K. Grauman. Learning a hierarchy of discriminative space-time neighborhood features for human action recognition. In CVPR, 2010.

[13] I. Laptev and T. Lindeberg. Space-time interest points. In ICCV 2003.

[14] I. Laptev, M. Marszałek, C. Schmid, and B. Rozenfeld. Learning realistic human actions from movies. In $C V P R$, 2008.

[15] C. Liu, J. Yuen, and A. Torralba. Nonparametric scene parsing: label transfer via dense scene alignment. In $C V P R$, 2009.

[16] J. Liu, J. Luo, and M. Shah. Recognizing realistic actions from videos in the wild. In CVPR, 2009.
[17] W.-C. Lu, Y.-C. F. Wang, and C.-S. Chen. Learning dense optical-flow trajectory patterns for video object extraction. In IEEE Conference on Advanced Video and Signal Based Surveillance, 2010.

[18] B. D. Lucas and T. Kanade. An iterative image registration technique with an application to stereo vision. In International Joint Conference on Artificial Intelligence, 1981.

[19] M. Marszałek, I. Laptev, and C. Schmid. Actions in context. In $C V P R, 2009$.

[20] P. Matikainen, M. Hebert, and R. Sukthankar. Trajectons: Action recognition through the motion analysis of tracked features. In ICCV workshop on Video-oriented Object and Event Classification, 2009.

[21] R. Messing, C. Pal, and H. Kautz. Activity recognition using the velocity histories of tracked keypoints. In ICCV, 2009.

[22] E. Nowak, F. Jurie, and B. Triggs. Sampling strategies for bag-of-features image classification. In ECCV, 2006.

[23] M. Rodriguez, J. Ahmed, and M. Shah. Action MACH: A spatio-temporal maximum average correlation height filter for action recognition. In $C V P R, 2008$.

[24] C. Schüldt, I. Laptev, and B. Caputo. Recognizing human actions: A local SVM approach. In ICPR, 2004.

[25] P. Scovanner, S. Ali, and M. Shah. A 3-dimensional SIFT descriptor and its application to action recognition. In ACM Multimedia, 2007.

[26] J. Shi and C. Tomasi. Good features to track. In CVPR, 1994.

[27] J. Sun, X. Wu, S. Yan, L.-F. Cheong, T.-S. Chua, and J. Li. Hierarchical spatio-temporal context modeling for action recognition. In $C V P R, 2009$.

[28] N. Sundaram, T. Brox, and K. Keutzer. Dense point trajectories by GPU-accelerated large displacement optical flow. In ECCV, 2010.

[29] G. W. Taylor, R. Fergus, Y. LeCun, and C. Bregler. Convolutional learning of spatio-temporal features. In ECCV, 2010.

[30] H. Uemura, S. Ishikawa, and K. Mikolajczyk. Feature tracking and motion compensation for action recognition. In BMVC, 2008.

[31] M. M. Ullah, S. N. Parizi, and I. Laptev. Improving bag-offeatures action recognition with non-local cues. In $B M V C$, 2010.

[32] H. Wang, M. M. Ullah, A. Kläser, I. Laptev, and C. Schmid. Evaluation of local spatio-temporal features for action recognition. In $B M V C, 2009$.

[33] G. Willems, T. Tuytelaars, and L. V. Gool. An efficient dense and scale-invariant spatio-temporal interest point detector. In ECCV, 2008.

[34] L. Yeffet and L. Wolf. Local trinary patterns for human action recognition. In ICCV, 2009.

[35] J. Yuan, Z. Liu, and Y. Wu. Discriminative subvolume search for efficient action detection. In CVPR, 2009.

[36] J. Zhang, M. Marszałek, S. Lazebnik, and C. Schmid. Local features and kernels for classification of texture and object categories: A comprehensive study. IJCV, 73(2):213-238, 2007. 